\title{
ON THE GAP BETWEEN TRIVIAL AND NONTRIVIAL INITIAL SEGMENT PREFIX-FREE COMPLEXITY
}

\author{
MARTIJN BAARTSE AND GEORGE BARMPALIAS
}

\begin{abstract}
An infinite sequence $X$ is said to have trivial (prefix-free) initial segment complexity if $K\left(X\left\lceil_{n}\right) \leq^{+} K\left(0^{n}\right)\right.$ for all $n$, where $K$ is the prefix-free complexity and $\leq^{+}$denotes inequality modulo a constant. In other words, if the information in any initial segment of it is merely the information in a sequence of 0 s of the same length. We study the gap between the trivial complexity $K\left(0^{n}\right)$ and the complexity of a non-trivial sequence, i.e. the functions $f$ such that

(*) $K\left(X \uparrow_{n}\right) \leq^{+} K\left(0^{n}\right)+f(n)$ for all $n$

for a non-trivial (in terms of initial segment complexity) sequence $X$. We show that given any $\Delta_{2}^{0}$ unbounded non-decreasing function $f$ there exist uncountably many sequences $X$ which satisfy $(\star)$. On the other hand there exists a $\Delta_{3}^{0}$ unbounded non-decreasing function $f$ which does not satisfy $(\star)$ for any $X$ with non-trivial initial segment complexity. This improves the bound $\Delta_{4}^{0}$ that was known from [CM06]. Finally we give some applications of these results.
\end{abstract}

\section{INTRODUCTION}

It is an interesting idea to try to express computability, or equivalently definability, in terms of initial segment complexity. Chaitin Cha76 did exactly this, when he proved that a set $X$ is computable iff its initial segments have minimal (plain) Kolmogorov complexity. Let $C$ denote the plain Kolmogorov complexity and $\leq^{+}$denote inequality modulo a constant. For all strings $\sigma$ we have $C(\sigma) \geq^{+} C\left(0^{|\sigma|}\right)={ }^{+} C(|\sigma|)$ (where $n=^{+} m$ if $n \leq^{+} m$ and $m \leq^{+} n$ ). Chaitin showed that a set $X$ is computable iff $C\left(X \uparrow_{n}\right) \leq^{+} C(n)$ for all $n \in \mathbb{N}$. Can we express or at least 'approximate' computability in terms of the prefix-free complexity $K$ ? Chaitin Cha76 showed that every set $X$ which has minimal prefix-free initial segment complexity, i.e. $K\left(X \uparrow_{n}\right) \leq^{+} K(n)$ for all $n \in \mathbb{N}$, is $\Delta_{2}^{0}$. The sets that satisfy this condition are called $K$-trivial and are known to form an interesting proper subclass of $\Delta_{2}^{0}$. Moreover there are non-computable $K$-trivial sets, so it seems impossible to characterize computability in terms of prefix-free complexity.

A natural question is, how large a margin can we allow above the minimal complexity $K(n)$ for the first $n$ bits of a set in the above condition so that we still get a considerable restriction on the class of sets that satisfies it? For example, we may ask if there is an unbounded non-decreasing function $f$ such that any set satisfying

$$
K\left(X \uparrow_{n}\right) \leq^{+} K(n)+f(n) \text { for all } n \in \mathbb{N}
$$

is $\Delta_{2}^{0}$, or even $K$-trivial.

Key words and phrases. Kolmogorov complexity, initial segment prefix-free complexity, $K$ triviality, low for $\Omega$. 
There is a constant $c$ such that $K(\sigma)+c \geq K\left(0^{|\sigma|}\right)=K(|\sigma|)$ for all strings $\sigma$. Hence, modulo a constant, $K(n)$ is a lower bound of the complexity of any string of length $n$. A set $X$ is $K$-trivial if it has the lowest possible initial segment complexity, namely $K\left(X \uparrow_{n}\right) \leq^{+} K(n)$. Suppose that we are given a non-decreasing unbounded function $f: \mathbb{N} \rightarrow \mathbb{N}$. It is plausible that based on $f$ one can construct a set $X$ which is not $K$-trivial but $K\left(X \uparrow_{n}\right) \leq^{+} K(n)+f(n)$ for all $n \in \mathbb{N}$. Intuitively, we would try to construct $X$ such that the complexity of its first $n$ bits increases when $f$ is sufficiently large. Since $\lim _{s} f(s)=\infty$ one would hope that we can achieve $\lim \left(K\left(X \uparrow_{n}\right)-K(n)\right)=\infty$ so that $X$ is not $K$-trivial.

Surprisingly, this is not the case. This was shown in [CM06] where an unbounded non-decreasing function $f$ was constructed such that for each set $X$,

$$
\text { If } K\left(X \uparrow_{n}\right) \leq^{+} K(n)+f(n) \text { for all } n \in \mathbb{N} \text {, then } X \text { is } K \text {-trivial. }
$$

Following [DH10, End of Section 10.12] an analysis of the proof shows that the function $f$ is $\Delta_{4}^{0}$. In [BV] it was shown that $f$ cannot be $\Delta_{2}^{0}$. In fact, it was shown that if $f$ is $\Delta_{2}^{0}$, unbounded and non-decreaing, then there exists a c.e. set $X$ which is not $K$-trivial but $K\left(A \uparrow_{n}\right) \leq^{+} K(n)+f(n)$ for all $n \in \mathbb{N}$. In Section 3 we use a result from $[\mathrm{BS}]$ in order to show that there is a $\Delta_{3}^{0}$ unbounded non-decreasing function $f$ satisfying 1.2 .

Theorem 1.1. There exists a $\Delta_{3}^{0}$ unbounded non-decreasing function $f: \mathbb{N} \rightarrow \mathbb{N}$ such that if $K\left(X \uparrow_{n}\right) \leq^{+} K(n)+f(n)$ for all $n \in \mathbb{N}$ and some set $X$ then $X$ is K-trivial.

The diagonalization employed in the proof of Theorem 1.1 (originally from CM06]) is particularly interesting since it deals with all possible sequences $X$. A discussion for cases where the oracles $X$ are restricted in a certain arithmetical class can be found in [BV], Section 5]. For example, the following facts were shown.

(a) There is a $\Delta_{2}^{0}$ function $f$ with $\lim _{n} f(n)=\infty$ such that 1.2 holds for all $\Sigma_{1}^{0}$ sets.

(b) There is no $\Delta_{2}^{0}$ unbounded nondecreasing function $f$ such that 1.2 holds for all $\Sigma_{1}^{0}$ sets.

(c) If $\lim _{n}(f(n)-K(n))=\infty$ then there are uncountably many infinite sequences $X$ satisfying 1.1 .

Notice that it is not clear whether (a) holds for an arithmetical class which is larger than $\Sigma_{1}^{0}$. In general, one can ask if given a $\Delta_{2}^{0}$ unbounded function $f$ one can construct a set $X$ which is not $K$-trivial but $K\left(X \uparrow_{n}\right) \leq^{+} K(n)+f(n)$ holds for all $n \in \mathbb{N}$.

In Section 4 we show that if the unbounded nondecreasing function which gives an upper bound on the excess complexity that the first $n$ bits of a sequence can have is $\Delta_{2}^{0}$, then there are continuum many sequences meeting this condition. This result contrasts (a) above.

Theorem 1.2. Let $g$ be a $\Delta_{2}^{0}$ unbounded nondecreasing function. There are uncountably many sets $X$ such that $K\left(X \uparrow_{n}\right) \leq+K(n)+g(n)$ for all $n \in \mathbb{N}$. In fact, there is a non-empty perfect $\Pi_{1}^{0}$ class which consists entirely of such sets $X$.

We would like to strengthen Theorem 1.2 so that the constructed $\Pi_{1}^{0}$ class does not have any $K$-trivial members. The reason for this is various applications that 
are based on basis theorems for $\Pi_{1}^{0}$ classes, as we explain below. Before we do this, it's worth considering if it is possible to obtain this strengthening without extra effort. In other words, if every perfect $\Pi_{1}^{0}$ class has a $\Pi_{1}^{0}$ subclass without $K$-trivial members. The answer is strongly negative. The following can be shown using standard methods in computability theory.

There is a perfect $\Pi_{1}^{0}$ class such that every $\Pi_{1}^{0}$ subclass of it has computable members.

However, with considerable effort, it is possible to modify the proof of Theorem 1.2 so that we get the following stronger result.

Theorem 1.3. The $\Pi_{1}^{0}$ class $P$ of Theorem 1.2 can be chosen such that it has no $K$-trivial members.

The value of this strengthening of Theorem 1.2 lies on the use of basis theorems for $\Pi_{1}^{0}$ classes in order to get sequences with certain computational properties with very low but non-trivial prefix-free complexity. We discuss this direction in Section 2. with a special attention on low for $\Omega$ sequences. Recall that $\Omega$ is the halting probability of a universal prefix-free machine. Also, given a Martin-Löf random sequence $Y$ we say that $X$ is low for $Y$ if $Y$ is Martin-Löf random relative to $X$.

Before we embark into a detailed discussion of our results, it seems appropriate to note a possible connection between our work and work on a different type of gap functions that were studied in BD09. For example they study functions $h: \mathbb{N} \rightarrow \mathbb{N}$ such that $K\left(X\left\lceil_{n}\right) \geq^{+} n-h(n)\right.$ is a sufficient condition for a set $X$ to be Martin-Löf random. These gap functions may refer to randomness or triviality, but are different than the ones that we study in this paper. Although we have not found a direct relation between these notions, there is an analogy in the two lines of research.

We finish this introduction with a word on terminology. In Sections 3,4 and 5 we use the notion of a tree in the Cantor space. This can be defined in the following two different ways:

(i) As a downward $\subseteq$-closed set of strings.

(ii) As a partial map from strings to strings, which preserves compatibility and incompatibility relations.

Perfect trees correspond to total maps in clause (ii). For convenience, in Section 3 we refer to the first formulation while in Section 4 we refer to the latter one. Level $n$ in a tree under (i) is the collection of strings of length $n$ which belong to the tree. On the other hand, if $T: 2^{<\omega} \rightarrow 2^{<\omega}, \sigma \rightarrow T_{\sigma}$ is a tree under (ii), level $n$ of $T$ refers to the collection of the strings $T_{\tau}$ such that $T_{\tau}$ is defined and $\tau$ has length $n$. If in any level of $T$ the strings have the same length (as will be the case in Section (4), this length is said to be the height of this level.

The weight of a prefix-free set $S$ of strings is defined to be the sum $\sum_{\sigma \in S} 2^{-|\sigma|}$. The weight of a prefix-free machine $M$ is defined to be the weight of its domain. Prefix-free machines are most often built in terms of request sets. A request set $L$ is a set of tuples $\langle\rho, \ell\rangle$ where $\rho$ is a string and $\ell$ is a positive integer. A 'request' $\langle\rho, \ell\rangle$ represents the intention of describing $\rho$ with a string of length $\ell$. We say that $L$ is a bounded request set if $\sum\left\{2^{-|\ell|} \mid \exists \rho,\langle\rho, \ell\rangle \in L\right\}<1$. This sum is the weight of the request set $L$. The Kraft-Chaitin theorem (see e.g. [DH10, Section 2.6]) says that for every bounded request set $L$ which is c.e., there exists a prefix-free machine $M$ such that for each $\langle\rho, \ell\rangle \in L$ there exists a string $\tau$ of length $\ell$ such that $M(\tau)=\rho$. 
Finally, it is appropriate to view the (prefix-free) initial segment complexity of a sequence $X$ as the function $n \rightarrow K\left(X \uparrow_{n}\right)$. In this way, for example, Theorem 1.1 can be concisely stated as 'There exists a $\Delta_{3}^{0}$ unbounded non-decreasing function such that any sequence whose initial segment complexity is $\leq^{+} K(n)+f(n)$ is $K$-trivial'.

\section{Applichtions of Theorems 1.2 and 1.3}

2.1. A gap between the finite and the uncountable. We are interested in the cardinality of the sequences with initial segment complexity $\leq c \cdot K(n)+d$, where $c \geq 1$ is a real number and $d \in \mathbb{N}$. By the coding theorem (see e.g. Nie09, Theorem 2.2.26]) if $c=1$ and $d$ is any integer there are finitely many such sequences. In this section we use Theorem 1.2 to show that for any $c>1$ there exists $d \in \mathbb{N}$ such that there are continuum many sequences with initial segment complexity $\leq c \cdot K(n)+d$. First we need the following.

Lemma 2.1. Let $c>1$ be a real number. There exists a $\Delta_{2}^{0}$ unbounded nondecreasing function $f$ such that $K(n)+f(n) \leq c \cdot K(n)$ for all $n \in \mathbb{N}$.

Proof. Let $q>0$ be a rational number such that $q+1<c$. Then $K(n)+q \cdot K(n) \leq$ $c \cdot K(n)$ for each $n \in \mathbb{N}$. Let $f(n)$ be the largest $t \in \mathbb{N}$ such that $t \leq q \cdot K(i)[s]$ for all $i \geq n$ and all $s \in \mathbb{N}$. Clearly $f$ is computable from $\emptyset^{\prime}$, hence $\Delta_{2}^{0}$. Moreover since $K(i)[s]$ is non-increasing in $s$, for each $n \in \mathbb{N}$ the number $f(n)$ is the largest $t$ such that $t \leq q \cdot K(i)$ for all $i \geq n$. Hence $f$ is non-decreasing. Since $\lim _{i} K(i)=\infty$ it is also unbounded. By the definition of $f$ we have $K(n)+f(n) \leq K(n)+q \cdot K(n) \leq$ $c \cdot K(n)$ for all $n \in \mathbb{N}$.

By combining Lemma 2.1 and Theorem 1.2 we have the following.

Corollary 2.2. Let $c>1$ be a real number. For some $d \in \mathbb{N}$ there exist uncountably many sequences $X$ with initial segment complexity $\leq c \cdot K(n)+d$.

Notice that Corollary 2.2 improves the basic fact (c) that was mentioned in Section 1 .

2.2. Complexity of low for $\Omega$ sequences. One advantage of obtaining an effective uncountable class in Theorems 1.2 and 1.3 is that we can use a variety of basis theorems in order to obtain sequences with certain computational properties and low but not trivial prefix-free complexity. For example, Theorem 1.3 shows that given any non-decreasing unbounded $\Delta_{2}^{0}$ function $g$ there is a computably dominated set whose initial segment prefix-free complexity is $\leq^{+} K(n)+g(n)$ but not $\leq^{+} K(n)$. Here we use the computably dominated basis theorem for $\Pi_{1}^{0}$ classes. Perhaps a more striking example is the following result about low for $\Omega$ sequences.

The applications in this section are focussed on the class of low for $\Omega$ sequences. Hence it is appropriate here to recall a few of the basic properties of this class. The low for $\Omega$ basis theorem says that every non-empty $\Pi_{1}^{0}$ class has a low for $\Omega$ member. It is an easy consequence of compactness and was shown in [RS10 and independently in DHMN05. Note that Theorem 2.4 below is a generalized version of the low for $\Omega$ basis theorem. All $K$-trivial sets are low for $\Omega$ by [HNS07]. In fact the $K$-trivial sets are the only $\Delta_{2}^{0}$ low for $\Omega$ sets. This follows from the fact that all $K$-trivial sets are computable from $\Omega$ and the results in [HNS07]. For a presentation see [Nie09, Theorem 8.1.18]. 
Corollary 2.3. Let $g$ be an unbounded non-decreasing $\Delta_{2}^{0}$ function. Then there exists a low for $\Omega$ sequence $X$ which is not $K$-trivial and $K\left(X \uparrow_{n}\right) \leq^{+} K(n)+g(n)$ for all $n \in \mathbb{N}$.

Proof. Consider the $\Pi_{1}^{0}$ class of Theorem 1.3 for the given $g$. By the low for $\Omega$ basis theorem it has a low for $\Omega$ member $X$. By the properties of the class, $X$ is not $K$-trivial and satisfies the desired inequality.

To obtain uncountably many sets as in Corollary 2.3 we need the following generalized version of the low for $\Omega$ basis theorem.

Theorem 2.4. Let $Z$ be a set and $X$ be $Z$-random. Every nonempty $\Pi_{1}^{0}(Z)$ class contains a nonempty $\Pi_{1}^{0}(Z \oplus X)$ subclass class which consists of low for $X$ sets.

Proof. Let $P$ be a $\Pi_{1}^{0}(Z)$ class and let $\left(U_{i}\right)$ be a universal oracle Martin-Löf test. For each $i \in \mathbb{N}$ let $V_{i}$ be the set of reals $A$ which are in $U_{i}^{Y}$ for all $Y \in P$. Clearly $\mu\left(V_{i}\right)<2^{-i}$ and by compactness $V_{i}$ is a $\Sigma_{1}^{0}(Z)$ class (uniformly in $i$ ). Therefore $\left(V_{i}\right)$ is a Martin-Löf test relative to $Z$. Since $X$ is random relative to $Z$, there is some $i_{0} \in \mathbb{N}$ such that $X \notin V_{i_{0}}$. This means that there are paths $Y$ in $P$ such that $X \notin U_{i_{0}}^{Y}$. Let us denote the collection of these sets by $Q$. Clearly $Q$ is a nonempty $\Pi_{1}^{0}(X \oplus Z)$ subclass of $P$. Since $\left(U_{i}\right)$ was chosen universal, for any path $Y \in Q$ the set $X$ is random relative to $Y$.

If we let $Z=\emptyset$ and $X=\Omega$ in Theorem 2.4 we get the following. Notice that by the basic facts that we discussed above, if the given $\Pi_{1}^{0}$ class does not have any $K$-trivial members then the $\Pi_{1}^{0}\left[\emptyset^{\prime}\right]$ subclass given by Corollary 2.5 has no $\Delta_{2}^{0}$ members.

Corollary 2.5. Every nonempty $\Pi_{1}^{0}$ class contains a nonempty $\Pi_{1}^{0}\left[\emptyset^{\prime}\right]$ subclass which consists entirely of low for $\Omega$ sets.

Now we are ready to argue for the following generalized version of Corollary 2.3 .

Corollary 2.6. Let $g$ be an unbounded non-decreasing $\Delta_{2}^{0}$ function. There exist uncountably many low for $\Omega$ sequences $X$ which are not $K$-trivial and $K\left(X \uparrow_{n}\right) \leq^{+}$ $K(n)+g(n)$ for all $n \in \mathbb{N}$.

Proof. Consider the $\Pi_{1}^{0}$ class of Theorem 1.3 for the given $g$. Then use Corollary 2.5 to obtain a non-empty subclass $P$ of it, which is $\Pi_{1}^{0}\left[\emptyset^{\prime}\right]$. Since the original class does not contain $K$-trivial sequences, so does $P$. As we recalled above, every $\Delta_{2}^{0}$ low for $\Omega$ sequence is $K$-trivial. Hence $P$ does not have $\Delta_{2}^{0}$ members. Since it is a $\Pi_{1}^{0}\left[\emptyset^{\prime}\right]$ class it follows that it is perfect, hence uncountable. Finally, its members satisfy the desired properties since it is a subclass of the original class.

We now wish to obtain a version of Corollary 2.3 for sequences that are not low for $\Omega$. We need a basis theorem for $\Pi_{1}^{0}$ classes that establishes the existence of sequences that are not low for $\Omega$. The following is the first step towards this basis theorem. We say that a countable class $\mathcal{C}=\subseteq 2^{\omega}$ is uniformly $\mathbf{0}^{\prime}$-computable if it can be presented as $\left\{\Phi_{f(e)}^{\emptyset^{\prime}} \mid e \in \mathbb{N}\right\}$, where $f$ is a computable sequence of indices which correspond to total $\emptyset^{\prime}$-computable functions.

Lemma 2.7. Let $T: 2^{<\omega} \rightarrow 2^{<\omega}$ be a perfect $\Delta_{2}^{0}$ tree and let $\mathcal{C} \subseteq 2^{\omega}$ be a uniformly $\mathbf{0}^{\prime}$-computable class. Then there is a $\Delta_{2}^{0}$ path of $T$ which is not in $\mathcal{C}$. 
Proof. Let $\mathcal{C}=\left\{\Phi_{f(e)}^{\emptyset^{\prime}} \mid e \in \mathbb{N}\right\}$, where $f$ is a computable function and $\Phi_{f(e)}^{\emptyset^{\prime}}$ is total for every $e \in \mathbb{N}$. Define a path $A$ inductively as follows. If $A\left\lceil_{n}=\sigma\right.$ is defined, let $A \uparrow_{n+1}$ be $\sigma * i$, where $i$ is chosen such that $T_{\sigma * i} \not \subset \Phi_{f(n)}^{\emptyset^{\prime}}$. Clearly $A$ and $T_{A}:=\cup_{\sigma \subset A} T_{\sigma}$ are $\Delta_{2}^{0}$ and $T_{A} \neq \Phi_{f(e)}^{\emptyset^{\prime}}$ for all $e \in \mathbb{N}$.

Now we can state a strong version of the promised basis theorem. Observe the contrast with the low for $\Omega$ basis theorem.

Corollary 2.8. Every perfect $\Delta_{2}^{0}$ tree contains a path which is not low for $\Omega$. In particular, there is no perfect $\Pi_{1}^{0}$ class containing only low for $\Omega$ paths.

Proof. Let $T \subseteq 2^{<\omega}$ be a perfect $\Delta_{2}^{0}$ tree. The $K$-trivial sets form a $\emptyset^{\prime}$-computable class by [Nie09, Theorem 5.3.28]. Apply Lemma 2.7 to get a $\Delta_{2}^{0}$ path $X$ of $T$ which is not $K$-trivial. As we discussed above, every $\Delta_{2}^{0}$ low for $\Omega$ set is $K$-trivial. Therefore the path $X$ of $T$ is not low for $\Omega$.

Finally we can show the following analogue of Corollary 2.3

Corollary 2.9. Let $g$ be an unbounded non-decreasing $\Delta_{2}^{0}$ function. Then there exists a sequence $X$ that is not low for $\Omega$ and $K\left(X \uparrow_{n}\right) \leq^{+} K(n)+g(n)$ for all $n \in \mathbb{N}$.

Proof. Consider the $\Pi_{1}^{0}$ class of Theorem 1.1 for the given $g$. Then use Corollary 2.8 to obtain a member $X$ of it which is not low for $\Omega$. Since $X$ is a member of the class, it satisfies the desired inequality.

\section{Proof of Theorem 1.1}

3.1. Preliminary facts. In this section we give a basic fact about the $K$-trivial sets, which is largely a consequence of the work done in BS]. First, we need the following 'uniformity' lemma.

Lemma 3.1. Given a $\emptyset^{\prime}$-computable sequence $\left(T_{i}\right)$ of trees with finitely many paths such that $T_{i}^{\prime \prime} \leq_{T} \emptyset^{\prime \prime}$ uniformly in $i$, there is a $\emptyset^{\prime \prime}$-computable function $f$ such that for each $i$ the number $f(i)$ is a code for a finite set of indices $t_{j}, j<k_{i}$ such that there are exactly $k_{i}$ paths through $T_{i}$ and these are $\Phi_{t_{j}}^{\emptyset^{\prime}}$ for $j<k_{i}$.

Proof. Given $i$ we show how to define $f(i)$ computably in $\emptyset^{\prime \prime}$. First we ask the cardinality $k_{i}$ of $\left[T_{i}\right]$. This can be decided in $\emptyset^{\prime \prime}$, see BS, Corollary 2.10]. Then we can search for $k_{i}$ incomparable strings $\sigma_{j}, j<k_{i}$ of the same length, such that for each $j<k_{i}$ the subtree of $T_{i}$ below $\sigma_{j}$ has a unique infinite path. By the definition of $k_{i}$ such strings exist. Moreover the check amounts to asking for a given string $\sigma$ if for all levels $\ell$ above $|\sigma|$ there exists a level $n>\ell$ such that there exists exactly one string of level $\ell$ which extends $\sigma$ and has an extension at level $n$.

This is a $\Pi_{2}^{0}$ question. Hence the condition can be checked computably in $T_{i}^{\prime \prime}$. Since $T_{i}^{\prime \prime} \leq_{T} \emptyset^{\prime \prime}$ (uniformly in $i$ ) the strings can be found computably in $\emptyset^{\prime \prime}$. Once we determine $\sigma_{j}, j<k_{i}$ we can effectively obtain the indices $t_{j}, j<k_{j}$ as follows. Given $j<k_{i}$ we let $t_{j}$ be the program that defines the unique path of $T_{i}$ extending $\sigma_{j}$. This definition is sound since given a $\Delta_{2}^{0}$ tree with a unique path we can effectively get a $\Delta_{2}^{0}$ definition of it from the tree.

For each $e \in \mathbb{N}$ fix $T_{e}$ to be the set of strings $\sigma$ such that $K\left(\sigma \uparrow_{i}\right) \leq K(i)+e$ for each $i \leq|\sigma|$. Clearly the trees $T_{e}, e \in \mathbb{N}$ are uniformly $\Delta_{2}^{0}$. Moreover for each $e \in \mathbb{N}$ 
the set $\left[T_{e}\right]$ consists of the finitely many $K$-trivial infinite sequences with constant $e$. By [BS, Corollary 3.4] there exists a uniformly c.e. sequence $\left(Q_{e}\right)$ of trees and a constant $c$ such that $\left[Q_{e}\right]=\left[T_{e}\right]$ and $Q_{e}$ (as a set of strings) is $K$-trivial with constant $2 e+c$ for each $e \in \mathbb{N}$. Moreover, given a constant via which a set $Q$ is $K$-trivial one can $\emptyset^{\prime \prime}$-effectively obtain a reduction $Q^{\prime \prime} \leq_{T} \emptyset^{\prime \prime}$ (see BS, Proposition 3.6]). If we combine these facts with Lemma 3.1 we obtain the following.

Proposition 3.2. There is a $\emptyset^{\prime \prime}$-computable function $f$ such that for each $i$ the number $f(i)$ is a code for a finite set of indices $t_{j}, j<k_{i}$ such that the $K$-trivial sequences with constant e are exactly the ones given by $\Phi_{t_{j}}^{\emptyset^{\prime}}$ for $j<k_{i}$.

Using Proposition 3.2 one can revisit the argument given in [CM06] and explicitly make sure that the function $f$ of 1.2 is $\Delta_{3}^{0}$. Instead we give a different, more direct presentation of this argument in the following section.

3.2. Construction of $f$ of Theorem 1.1. Let us denote by $\mathcal{K}_{e}$ the class of $K$ trivial sequences with constant $e$. In the argument below we freely use the fact that:

$$
\text { Given } X \leq_{T} \emptyset^{\prime} \text { and } e \in \mathbb{N} \text { we can } \emptyset^{\prime \prime} \text {-computably decide if } X \in \mathcal{K}_{e} \text {. }
$$

Here the set $X$ is given in the sense of a reduction of it to $\emptyset^{\prime}$. We define an increasing sequence $\left(n_{k}\right)$ and let $f(t)$ be the least $k$ such that $n_{k} \geq t$. Given $k$ define $n_{k}>n_{k-1}$ to be the least number such that for each $e \leq k$ :

- For all $X \in \mathcal{K}_{e+k+2}-\mathcal{K}_{e}$ there exists $i<n_{k}$ such that $K\left(X \uparrow_{i}\right)>K(i)+e$.

- If $k>e+1$ and for some set $X$ the least number $i$ such that $K\left(X \uparrow_{i}\right)>$ $K(i)+e$ is in $\left[n_{k-2}, n_{k-1}\right)$ then there exists $j<n_{k}$ such that $K\left(X \curlyvee_{j}\right)>$ $K(j)+e+k$.

By Proposition 3.2 and (3.1) using $\emptyset^{\prime \prime}$ we can determine a large enough $n_{k}$ satisfying the first condition. For the second condition, notice that by the previous step (the definition of $\left.n_{k-2}\right)$ if the least number $i$ such that $K\left(X \uparrow_{i}\right)>K(i)+e$ is in $\left[n_{k-2}, n_{k-1}\right)$ then we have that $X \notin \mathcal{K}_{e+k}$. Hence for each such set, the string $X \uparrow_{n_{k-1}}$ is not extendible in the tree $T_{e+k}$. Hence by König's lemma there exists a level $\ell$ in $T_{e+k}$ at which no extendible string has $K\left(\sigma \uparrow_{i}\right)>K(i)+e$ for $i \in$ $\left[n_{k-2}, n_{k-1}\right)$. This level $\ell$ can be calculated using $\emptyset^{\prime}$ and is lower bound for $n_{k}$ satisfying the second condition. This concludes the definition of $\left(n_{k}\right)$ and shows that $f \leq_{T} \emptyset^{\prime \prime}$.

Now suppose that some set $X$ satisfies $K\left(X \curlyvee_{n}\right) \leq K(n)+f(n)+e$ for some $e>1$ and all $n \in \mathbb{N}$. For a contradiction, suppose that $X$ is not $K$-trivial. So let $t$ be the least number $>n_{e}$ such that $K\left(X \uparrow_{t}\right)>K(t)+e$. Let $k$ be such that $t \in\left[n_{k-2}, n_{k-1}\right)$. Then by the second condition of the definition of $n_{k}$ there exists some $j<n_{k}$ such that $K\left(X \uparrow_{j}\right)>K(j)+e+k$. But this contradicts the fact that $K\left(X \uparrow_{j}\right) \leq K(j)+f(j)+e$ since $f(j)<k$. This concludes the proof that $f$ satisfies 1.2 and is $\Delta_{3}^{0}$.

\section{Proof of Theorem 1.2}

It was observed in [BV, Section 5] that if $g$ is a $\Delta_{2}^{0}$ non-decreasing unbounded function, then there exists an unbounded non-decreasing function $f$ such that $f(n) \leq g(n)$ for all $n \in \mathbb{N}$ and it is approximable from above in the following 
way.

There is a computable approximation $f[s] \rightarrow f$ with $n \rightarrow f(n)[0]$ being the identity, $f(i)[s] \leq f(j)[s]$ for all $i<j$, and for each $s$ there exists a unique $n$ such that $f(n)[s] \neq f(n)[s+1]$, in which case $f(n)[s+1]=$ $f(n)[s]-1$.

Therefore we may replace $g$ with $f$ in the statement of the Theorem 1.2 The parameters $f(n)[s]$ can be viewed as movable markers that can only move from right to left and their initial position is $n$. Moreover by 4.1 at most one marker can move at each stage, and each marker can only move by one position (i.e. decrease its value by 1$)$. Now it suffices to define a perfect $\Pi_{1}^{0}$ tree $T$ and a prefix-free machine $M$ such that $K_{M}\left(X \uparrow_{n}\right) \leq^{+} K(n)+f(n)$ for all $n \in \mathbb{N}$ and all $X \in[T]$. Equivalently, its suffices to ensure that at each stage $s$

$$
K_{M}(\sigma) \leq K(|\sigma|)[s]+f(|\sigma|)[s] \text { for all } \sigma \text { on } T[s] \text { with }|\sigma| \leq s .
$$

4.1. Building the $\Pi_{1}^{0}$ class of Theorem 1.2. We define an effective sequence of $1-1$ maps $T[s]: 2^{<\omega} \rightarrow 2^{<\omega}$ which preserve the ordering and compatibility relations. These can be viewed as uniformly computable perfect trees, and we can consider the set of infinite paths through them:

$$
[T[s]]=\left\{X \mid \forall n \exists \sigma\left(|\sigma|=n \wedge T_{\sigma}[s] \supseteq X\lceil n)\right\}\right.
$$

which is a $\Pi_{1}^{0}$ class. We will also ensure that $[T[s+1]] \subseteq[T[s]]$ for each $s \in \mathbb{N}$ and that $T_{\sigma}=\lim _{s} T_{\sigma}[s]$ exists for each $\sigma \in 2^{<\omega}$. Then the downward closure of the range of the map $T$ is a $\Pi_{1}^{0}$ tree $T$ and $[T]=\cap_{s}[T[s]]$ is a perfect $\Pi_{1}^{0}$ class, where $T$ is the limit map $\sigma \rightarrow T_{\sigma}$. Level $n$ of tree $T[s]$ consists of the nodes $T_{\sigma}[s]$ for $\sigma \in 2^{n}$.

Intuitively, the above formal description amounts to starting from a certain map $\sigma \rightarrow T_{\sigma}[0]$ and at each stage $s>0$ moving the markers $T_{\sigma}[s-1]$ to possibly new positions (i.e strings) $T_{\sigma}[s]$. The movement of the markers $T_{\sigma}$ will satisfy the following conditions at each stage.

(i) The map $\sigma \rightarrow T_{\sigma}[0]$ is the identity.

(ii) Compatibility and incompatibility relations are preserved.

(iii) Each new position is the position of some marker at the previous stage.

(iv) All nodes of a level of $T$ have the same length.

(v) If some node $T_{\sigma}$ moves, then all nodes of the same or larger levels move.

(vi) If a level of $T$ moves at stage $s$, it moves to a number $\geq s$.

In order to meet 4.2 it suffices to control the enumeration of $M$-descriptions at stage $s$ of the construction by the following clause.

For all strings $\sigma$ on $T[s]$ of length $\leq s$ such that $K_{M}(\sigma)[s-1]>$ $K(|\sigma|)[s]+f(|\sigma|)[s]$ request an $M$-description of $\sigma$ of length $K(|\sigma|)[s]+f(|\sigma|)[s]$.

Let $n_{-1}[s]=0$ and for each $k \in \mathbb{N}$ let $n_{k}[s]$ be the least number such that $f\left(n_{k}\right)[s]>$ $2 k$ and $n_{k}[s]>n_{k-1}[s]$. By (4.1) we have $n_{k}[0]=2 k+1$. 
4.2. Ideal scenario. Suppose that the approximation $f[s]$ to $f$ was constant (hence $f$ was computable). Then each $n_{k}[s]$ would also be constant in $s$ and $k \rightarrow n_{k}$ would be computable. In that case it would suffice to let $T$ be any computable tree such that $T_{\sigma}$ has length $n_{|\sigma|}$ (in particular, the markers $T_{\sigma}$ do not move). Indeed, in that case the weight of the domain of $M$ would be at most

$$
\sum_{k} \sum_{|\sigma|=k}\left(\sum_{i=\left|T_{\sigma}-\right|}^{\left|T_{\sigma}\right|-1} u_{i} \cdot 2^{-f(i)}\right) \leq \sum_{k} 2^{k}\left(\sum_{i=n_{k-1}}^{n_{k}-1} u_{i} \cdot 2^{-2 k}\right) \leq \sum_{i} u_{i}<1
$$

where $\sigma^{-}$is the predecessor of $\sigma$ and $u_{i}$ is the weight of the descriptions of $i$ of the universal machine ${ }^{1}$ Also if $\sigma$ is the empty sequence then (by convention) $T_{\sigma^{-}}$ is the empty sequence. In this ideal scenario, each node $T_{\sigma}$ is responsible for the weight of the $M$-descriptions that are issued for strings between $T_{\sigma^{-}}$and $T_{\sigma}$. By the choice of $k \rightarrow n_{k}$ and since $\left|T_{\sigma}\right|=n_{|\sigma|}$ we have that the weight for which $T_{\sigma}$ is responsible is at most $2^{-|\sigma|} q_{\sigma}$, where $q_{\sigma}$ is the weight of the $U$-descriptions of numbers between $\left|T_{\sigma^{-}}\right|$and $\left|T_{\sigma}\right|$. Since for each $k$ there are $2^{k}$ nodes $T_{\sigma}$ with $|\sigma|=k$ it follows that the total weight of all $M$-descriptions is bounded. This way of making nodes responsible for certain weight of $M$-descriptions will be useful in Section 4.3 .

4.3. Real scenario. We will modify the argument of Section 4.2 to deal with the real possibility that the 'markers' $f(k)[s]$ may move to smaller numbers during the stages $s$. We will allow the revision of the positions of the markers $T_{\sigma}$ which define the tree $T$ as discussed above. The movement of the markers $T_{\sigma}$ and the enumeration of $M$ will follow the prescriptions given in Section 4.1, as well as the following condition.

(4.4) At each stage $s$ the height of the $k$ th level of $T[s]$ is $\geq n_{k}[s]$ for $k<s$.

Notice that 4.4 was implicit in Section 4.2. Now the main challenge is to bound the weight of the domain of $M$. The weight of the requested $M$-descriptions will be distributed to the markers $T_{\sigma}$ as follows. Each $T_{\sigma}$ is responsible for

(a) The $M$-descriptions of strings between the final position of $T_{\sigma^{-}}$and the final position of $T_{\sigma}$.

(b) The $M$-descriptions of strings $\rho$ which were on $T[s]$ at some stage $s$, $\rho \supset T_{\sigma}[s]$ but at $s+1$ level $|\sigma|$ was the least to move, and moved so that $\rho$ is no longer on $T[s+1]$.

Before we state the actual construction, we show that in any construction of $\sigma \rightarrow T_{\sigma}[s]$ which satisfies (i)-(vi) of Section 4.1. condition (4.4) and any enumeration of a prefix-free machine $M$ which is defined according to 4.3 the weight of the requests for $M$-descriptions is bounded.

Lemma 4.1. If a computable sequence of maps $\sigma \rightarrow T_{\sigma}[s]$ meets conditions (i)-(vi) of Section 4.1 and condition (4.4), then a prefix-free machine $M$ that is enumerated according to (4.3) has bounded domain.

\footnotetext{
${ }^{1}$ Notice that the final bound holds even if we merely defined $n_{k}$ to be the least number such that $f\left(n_{k}\right)>k$. The stronger condition $f\left(n_{k}\right)>2 k$ that we required in the definition of $n_{k}$ will be useful in the main argument, in Section 4.3
} 
Proof. Let $A_{\sigma}$ contain the $M$-descriptions under clause (a) above. Notice that $A_{\sigma}$ is empty unless $T_{\sigma^{-}}$reaches a limit. Also notice that the sets $A_{\sigma}$ may not be uniformly (in $\sigma$ ) computably enumerable, although $T_{\sigma}[s]$ is uniformly computable in $\sigma$ and $s$. Let $B_{\sigma}$ be the c.e. set of $M$-descriptions that are attributed to $T_{\sigma}$ under clause (b). Notice that any $M$-description that is issued must either fall under clause (a), or under clause (b). Hence it suffices to show that the sets $\cup_{\sigma} A_{\sigma}$ and $\cup_{\sigma} B_{\sigma}$ (where $\sigma$ ranges over all strings) have bounded weight.

By (4.4), the argument of Section 4.2, the definition of $k \rightarrow n_{k}[s]$ and the definition of $M$ by 4.3 shows that the weight of $\cup_{\sigma} A_{\sigma}$ is bounded. For $\cup_{\sigma} B_{\sigma}$ fix a string $\sigma$. As in Section 4.1 let $u_{i}$ be the weight of the descriptions of $i$ of the universal machine. Let $s_{1}, s_{2}, \ldots$ be the stages (finitely many or infinitely many) where enumerations into $B_{\sigma}$ occurred and $s_{0}=0$. These are typically the stages where level $|\sigma|$ was the least to move. Fix $i>0$. Following the calculation of Section 4.2 adapted to the snapshot at stage $s$ of the restriction of $T$ on extensions of $\sigma$, the weight of the descriptions that where enumerated in $B_{\sigma}$ at $s_{i}, i>0$ is at most $2^{-2|\sigma|} \cdot \sum_{j=s_{i-1}}^{s_{i}-1} u_{j}$. In this calculation we use 4.4 , property (vi) and the fact that at stage $s$ only strings of length $\leq s$ may have $M$-descriptions. Hence

$$
\operatorname{wgt}\left(B_{\sigma}\right) \leq \sum_{i}\left(2^{-2|\sigma|} \cdot \sum_{j=s_{i-1}}^{s_{i}-1} u_{j}\right) \leq 2^{-2|\sigma|} \cdot \sum_{i} u_{i}<2^{-2|\sigma|} .
$$

Since there are $2^{i}$ strings of length $i$ we have

$$
\operatorname{wgt}\left(\cup_{\sigma} B_{\sigma}\right) \leq \sum_{i}\left(\sum_{\sigma \in 2^{i}} \operatorname{wgt}\left(B_{\sigma}\right)\right) \leq \sum_{i}\left(\sum_{\sigma \in 2^{i}} 2^{-2|\sigma|}\right) \leq \sum_{i} 2^{-i} \leq 2 .
$$

Since both $\cup_{\sigma} A_{\sigma}$ and $\cup_{\sigma} B_{\sigma}$ have bounded weight, so does the domain of $M$.

Now we are ready to give the formal construction of $\sigma \rightarrow T_{\sigma}[s]$ and $M$ and verify the desired properties.

4.4. Construction. At stage $s+1$ do the following:

(I) If there is some $k \leq s$ such that $n_{k}[s+1]>n_{k}[s]$, pick the least one and let $t$ be the least number $>n_{k}[s+1]$ such that the $t$-th level of $T[s]$ is $>s$ (i.e. $\left|T_{\rho}[s]\right|>s$ if $|\rho|=t$ ). Then move level $k$ of $T$ to the current level $t$ as follows. For each $\sigma$ of length $k$ let $T_{\sigma}[s+1]$ equal to $T_{\sigma^{*}}[s]$ where $\sigma^{*}=\sigma * 0^{t-k}$. Also, let $T_{\sigma * \eta}[s+1]=T_{\sigma^{*} * \eta}[s]$ for all strings $\eta$.

(II) For all strings $\sigma$ on $T[s+1]$ of length $<s$, if $K_{M}(\sigma)[s]>K(|\sigma|)[s+$ $1]+f(|\sigma|)[s+1]$ request an $M$-description of $\sigma$ of length $K(|\sigma|)[s+1]+$ $f(|\sigma|)[s+1]$.

4.5. Verification. It is clear that the sequence of maps $\sigma \rightarrow T_{\sigma}[s]$ that we define in the construction meets conditions (i)-(vi) of Section 4.1. Moreover it satisfies (4.4) and the machine $M$ is enumerated according to 4.3 . By Lemma 4.1 the requested $M$-descriptions have bounded weight. Hence by the Kraft-Chaitin theorem there is a prefix-free machine that gives descriptions as requested in the construction. We express this fact by saying that $M$ is a prefix-free machine.

Clause (II) of the construction explicitly ensures 4.2 . It remains to show that the markers $T_{\sigma}[s]$ reach a limit. i.e. they are eventually permanently defined. We do this by induction on the levels of the trees $T[s]$. By the hypothesis on $f$ and the definition of $n_{k}[s]$, for each $k$ the sequence $\left(n_{k}[s]\right)_{s \in \mathbb{N}}$ converges. Let $t_{k}$ be the 
modulus of convergence of $\left(n_{k}[s]\right)_{s \in \mathbb{N}}$. We show that for each $n \in \mathbb{N}$, level $n$ of $T[s]$ reaches a limit with respect to $s$. By construction level 0 of $T$ will reach a limit by stage $t_{0}$. Assume that all levels $<k$ have reached a limit by stage $m$ and $m_{*}=\max \left\{m, t_{k}\right\}$. By construction level $k$ of $T$ will reach a limit by stage $m_{*}$. This concludes the induction step and the verification of the construction.

\section{Proof of Theorem 1.3}

A perfect $\Pi_{1}^{0}$ class is constructed by introducing 'splits' along every path in the class. That is, we make sure that each path splits into two paths at infinitely many lengths. In order to construct a $\Pi_{1}^{0}$ class which does not contain any $K$-trivial paths one has to introduce 'clumps' in the tree instead of mere splits. By choosing the 'clumps' large enough, we can be sure that they contain strings of appropriately high Kolmogorov complexity. This is based on the following well known and widely used fact (e.g. see [Bar10a, Theorem 2.6] or [DG08]).

There exists a computable function $f(e, n)$ such that for all $e, n \in$ $\mathbb{N}$ and any string $\sigma$ of length $n$ there exists an extension $\tau$ of $\sigma$ of length $f(e, n)$ such that $K\left(\tau \uparrow_{i}\right)>K(i)+e$ for some $i<|\tau|$.

Hence removing the ones that have low complexity (a computably enumerable event) leaves us with a non-empty class with the desired property. However this rather crude method is not compatible with the argument of Section 4 . Indeed, in that argument the nodes $T_{\sigma}$ of the constructed $\Pi_{1}^{0}$ tree $T$ were given as limits of their computable approximations $T_{\sigma}[s]$. If we tried to implement a strategy based on (5.1) we would have to ask for larger and larger 'clumps' above $T_{\sigma}$, each time this marker moves. Hence the sums in the calculations of Section 4 would no longer be bounded, even if one considers modifications of the function $k \rightarrow n_{k}$.

The solution is a more dynamic approach which is compatible with keeping the size of the 'clumps' above each movable node constant. We present the argument as an extension of the proof of Section $4^{2}$ In particular, we define a computable sequence of trees $\sigma \rightarrow T_{\sigma}[s]$ that satisfy (i)-(vi) of Section 4. Moreover in order to ensure 4.2 we build a machine $M$ which operates according to (4.3). Since we wish to obtain a $\Pi_{1}^{0}$ class with no $K$-trivial paths, we will enumerate a c.e. set $Q$ of strings such that

$$
[T]-[Q] \text { is non-empty and does not contain any } K \text {-trivial sequence }
$$

where for a set of strings $S$ we let $[S]=\{X \mid \exists \sigma \in S, \sigma \subset X\}$. As we explained in Section 4 the set of infinite paths $[T]$ of $T$ will be a $\Pi_{1}^{0}$ class. Hence $[T]-[Q]$ is a $\Pi_{1}^{0}$ class.

5.1. Additional requirements and strategy. To make sure that $[T]-[Q]$ does not contain any $K$-trivial sequences we add a set of parameters $\ell_{e}$ to the construction of Section 4 and satisfy the following additional requirements for all $e \in \mathbb{N}$.

$$
\begin{aligned}
& \text { If a string } \sigma \text { on level } \ell_{e+1} \text { of } T \text { has an extension in } \\
& {[T]-[Q] \text { then } K\left(\left.\sigma\right|_{i}\right)>K(i)+e \text { for some } i<|\sigma| \text {. }}
\end{aligned}
$$

Intuitively, (5.3) says that by level $\ell_{e+1}$ all paths of $T$ have been 'revealed' to be not $K$-trivial with constant $e$. In order to satisfy these conditions we will need to

\footnotetext{
${ }^{2}$ Alternatively one can construct a suitable $\Pi_{1}^{0}$ class as an effectively closed set in the Cantor space (e.g. see [BLS08, Theorem 7]) or as the set of extensions of a 0-1 partial computable function (e.g. see [BLS08, Theorem 9]).
} 
build an additional prefix-free machine $N$ in order to gain partial control of $K(t)$, $t \in \mathbb{N}$ (i.e. establish certain inequalities of the type $K(t)<m$ ). By the recursion theorem we may use the index $c>0$ of $N$ in our construction. Then

$$
K(t) \leq K_{N}(t)+c \text { for all } t \in \mathbb{N} .
$$

For each $e$ we have a strategy such that all these strategies together will make sure that the requirements of 5.3 are fulfilled. These strategies enumerate strings into $Q$ and requests into $N$ as follows. Let $\ell_{0}=0$ and $\ell_{e+1}=\ell_{e}+2 e+c+3$. Thus every string at level $\ell_{e}$ of the tree $T$ has $2^{2 e+c+3}$ extensions at level $\ell_{e+1}$. Moreover this holds at every stage $s$. A basic feature of the construction (that was not present in the argument of Section 4 4 is that only levels $\ell_{e}$ can cause changes in the approximation to the tree.

Each strategy for 5.3 works in cycles. A cycle of the strategy corresponding to $e$ will be interrupted upon a movement of a marker-node $T_{\eta}$ for $|\eta| \leq \ell_{e}$. Notice that this is an interaction of the strategy with the original construction of Section 4. Such events may be considered as injuries of the strategy. We will make sure that for each strategy for 5.3 they occur finitely often. The strategy corresponding to $e$ is committed to keep the weight of the $N$-requests that it issues to at most $2^{-e-2}$. In order to keep track of the weight that it adds in the domain of $N$ with its requests, the strategy has a parameter $b_{e}[s]$. We let $b_{e}[0]=e+4$. Each time the strategy is injured, the value of $b_{e}$ increases by 1 . Let $t_{e}[s]$ be the height of level $\ell_{e}$ on $T[s]$ plus $2 e+c+3$. Notice that the height of level $\ell_{e+1}$ is $\geq t_{e}[s]$.

A cycle of the strategy corresponding to $e$ starts at a stage $s+1$ by ensuring that $K\left(t_{e}[s]\right)<b_{e}[s]+c$. It does this by enumerating an $N$-description of $t_{e}[s]$ of length $b_{e}[s]$. It continues as follows, as long as the strategy is not injured. If $K(\eta)[t] \leq b_{\sigma}[s]+c+e$ at some later stage $t$ for some $\eta$ of length $t_{e}[s]$ on $T[s]$, it enumerates $\eta$ into $Q$ unless it is the last extension on $T[s]$ with that length of a node of $T[s]$ of level $\ell_{e}$ such that $[\eta] \cap[T[s]]-[Q[s]] \neq \emptyset$. In the latter event the cycle finishes. When the cycle finishes at some stage $k$, the strategy starts a new cycle by moving the nodes $T_{\sigma}$ with $|\sigma|=\ell_{e}$ to strings on $T[k]$ of length $>k$ that do not have an initial segment in $Q[k]$ (unless $T_{\sigma}[s]$ already had a prefix in $Q[s]$ ). This is possible since at each stage $s$ during the cycle, each string of level $\ell_{e}$ that does not have a prefix in $Q[s]$ has an infinite extension in $[T[s]]-[Q[s]]$.

Notice that there are three possible outcomes for a cycle of a sub-strategy.

(a) The cycle may be interrupted by injury.

(b) The cycle may finish.

(c) The cycle may never finish or be injured.

5.2. Calculating the $\boldsymbol{N}$-weight that is produced by a strategy. We wish to obtain an upper bound on the weight of the $N$-requests that a strategy issues in the course of the construction. Every such request is issued during a cycle of the strategy. Moreover exactly one request is issued within a cycle of the strategy. First we consider the requests that were issued in a cycle that was either injured or never finished. In the latter case, note that no more cycles will occur. Since the parameter $b_{e}$ increases by 1 upon each injury, we have the following bound on the $N$-weight that is attributed to the cycles that were either injured or never finished.

$$
\sum_{j} 2^{-b_{e}[0]-j}=\sum_{j} 2^{-e-4-j}=2^{-e-3} .
$$


For the calculation of the weight of the requests that were issued during a cycle which finished we have to argue in a different way. If such a cycle begins at stage $s+1$, it adds weight $2^{-b_{e}[s]}$ to the weight of the domain of $N$ and by the end of it at least $2^{2 e+c+3}$ strings of length $t_{e}[s]$ have $U$-descriptions of length $\leq b_{e}[s]+c+e$. In other words, for each such increase on the weight of $N$ we can count an increase in the domain of $U$ which is $2^{e+3}$ times larger. Since wgt $(U)<1$ the total weight of the requests that are issued during such cycles is bounded by $2^{-e-3}$. Adding the two kinds of $N$-weight increases, we get the following.

The weight of the $N$-requests of the strategy corresponding to $e$ is at most $2^{-e-2}$.

Hence $\sum_{e} 2^{-e-2}=2^{-1}$ is a bound for the total weight of the requests of $N$ that are produced in the construction.

5.3. Construction. We use the definitions and conventions of the argument of Section 4 For example, the map $\sigma \rightarrow T_{\sigma}[0]$ is the identity. Let $b_{e}[0]=e+4$ and $t_{e}[0]$ be the height of level $\ell_{e}$ in $T[0]$ plus $2 e+c+3$. At stage $s+1$, by "move level $\ell_{e}$ to level $t$ " we mean the following.

For each $\sigma$ of length $\ell_{e}$ choose the leftmost extension $\rho_{\sigma}$ of $\sigma$ of length $t$ such that either $T_{\sigma}[s]$ has a prefix in $Q[s+1]$ or $T_{\rho_{\sigma}}[s]$ does not have a prefix in $Q[s+1]$. Let $T_{\sigma}[s+1]=T_{\rho_{\sigma}}[s]$. Also, let $T_{\sigma * \eta}[s+1]=T_{\rho_{\sigma * \eta}}[s]$ for all strings $\eta$. For all $i>e$ let $b_{i}[s+1]=b_{i}[s]+1$ and for all $i \geq e$ let $t_{i}[s+1]$ be the height of level $\ell_{e}$ in $T[s+1]$ plus $2 e+c+3$.

The strategy corresponding to $e$ requires attention at some stage $s+1$ when there is an extension $\eta$ of length $t_{e}[s]$ of $T_{\sigma}[s]$ for some $\sigma$ of length $\ell_{e}$ with the property $K(\eta)[s] \leq b_{\sigma}[s]+c+e$ and $\eta \notin Q[s]$.

At even stages $s+1$ do the following:

(EI) Ensure (4.4). If there is some $k \leq s$ such that $n_{k}[s+1]>n_{k}[s]$, pick the least one and let $t$ be the least number $\geq n_{k}[s+1]$ such that $\left|T_{\rho}[s]\right|>s$ for all $\rho$ of length $t$. Let $e$ be the largest number such that $\ell_{e} \leq k$. Then move level $\ell_{e}$ to level $t$ and set $b_{e}[s+1]=b_{e}[s]+1$.

(EII) Enumerate requests into $M$. For all strings $\sigma$ on $T[s+1]$ of length $\leq s$, if $K_{M}(\sigma)[s]>K(|\sigma|)[s+1]+f(|\sigma|)[s+1]$ request an $M$-description of $\sigma$ of length $K(|\sigma|)[s+1]+f(|\sigma|)[s+1]$.

At odd stages $s+1$ do the following

(OI) Enumerate requests into $N$. For all $e$ such that $t_{e}[s] \leq s$ and no $N$ description of $t_{e}[s]$ of length $b_{e}[s]$ has been enumerated yet, enumerate an $N$-description of $t_{e}[s]$ of length $b_{e}[s]$.

(OII) Enumerate into $Q[s+1]$. Let $e$ be the least number such that $t_{e}[s] \leq s$ for which the strategy corresponding to $e$ requires attention. If no such $e$ exists, end this stage. Otherwise let $Q_{*}$ be the union of $Q[s]$ and the strings $\eta$ of length $t_{e}[s]$ in $T[s]$ with the property $K(\eta)[s] \leq b_{e}[s]+c+e$. If for each $\sigma$ of length $\ell_{e}$

either $T_{\sigma}[s]$ has a prefix in $Q_{*}$ or $\left[T_{\sigma}[s]\right] \cap[T[s]]-\left[Q_{*}\right] \neq \emptyset$

let $Q[s+1]=Q_{*}$. If not, let $P$ be the set of strings $\nu$ of level $\ell_{e+1}$ that are the leftmost extension of $T_{\sigma}[s]$ for some $\sigma$ with $|\sigma|=\ell_{e}$ such that either $T_{\sigma}[s]$ has a prefix in $Q_{*}$ or $[T[s]] \cap[\nu]-\left[Q_{*}\right] \neq \emptyset$. Let $Q[s+1]=Q_{*}-P$, 
let $t$ be the least number such that the height of level $t$ is $>s$ and move level $\ell_{e}$ to level $t$.

5.4. Verification. In order to make a precise application of the recursion theorem, at this point we may view $c$ as an arbitrary parameter of the construction (not necessarily an index of $N$ ).

A basic feature of the construction is that for all $e$, all $\sigma$ of length $\ell_{e}$ and all stages $s$, either $T_{\sigma}[s]$ has a prefix in $Q[s]$ or $\left[T_{\sigma}[s]\right] \cap[T[s]]-[Q[s]] \neq \emptyset$. This property holds at stage 0 since $Q[0]=\emptyset$ and is preserved inductively throughout the construction via Step OII, Step EI and (5.6). In particular, (5.6) is justified as a way of moving the nodes of the tree (i.e. the conditions that it asks for the new positions of the nodes can be satisfied). As a consequence, since we never enumerate any prefix of $T_{\emptyset}$ into $Q$, we have $[T]-[Q] \neq \emptyset$.

Now one may view the construction as a computable function which takes $c$ and returns a program for $N$ (or rather the request set associated with $N$ ). By the recursion theorem we may choose $c$ to be an index of $N$. After these necessary justifications we verify the desired properties of the set nonempty $[T]-[Q]$ in a series of lemmas.

Lemma 5.1. There is a prefix-free machine $M$ with the specification given in the construction.

Proof. According to the justification above, the construction defines a computable sequence of maps $\sigma \rightarrow T_{\sigma}[s]$. A simple inspection of the construction shows that this sequence meets conditions (i)-(vi) of Section 4.1. The same holds for condition 4.4 restricted to the even stages (since only at even stages descriptions are enumerated into $M$ this restriction is allowed). The request set for the prefix-free machine $M$ that is enumerated in the construction follows 4.3 . By Lemma 4.1 the requests for $M$ have bounded weight. This shows that the specification of $M$ given in the construction corresponds to an actual prefix-free machine.

Lemma 5.2. There is a prefix-free machine $N$ with the specification given in the construction.

Proof. The argument of Section 5.2 applies to the construction and shows that the weight of the requests for $N$ is finite. The lemma follows by the Kraft-Chaitin theorem.

Notice that the markers $T_{\sigma}$ may move for two reasons. One is the original strategies of Section 4 (Clause (EI) of the construction) and the other is the additional strategies (Clause (OII) of the construction). The reason that the first movement stops is that the approximations to $f$ converge. The second movement stops because the additional strategy corresponding to a level can only conclude a certain number of cycles, as we argued in Section 5.2. The proof of the following fact requires the combination of these arguments, in an induction.

Lemma 5.3. Each movable marker $T_{\sigma}$ reaches a limit.

Proof. If levels move then the least level to move is level $\ell_{e}$ for some $e$, so it suffices to show that all levels $\ell_{e}$ reach a limit. Inductively assume that by stage $s_{0}$ all markers $T_{\eta}$ with $|\eta|<\ell_{e}$ have reached a final value. We show that all markers $T_{\rho}$ for $|\rho|=\ell_{e}$ reach a limit by some later stage. Let $s_{1}>s_{0}$ be a stage after which $n_{\ell_{e}}$ remains constant. 
After stage $s_{1}$ the strategy corresponding to $e$ will not be 'injured'. In terms of the analysis of Section 5.1. each time it moves after stage $s_{1}$ it completes a cycle, while $b_{e}$ remains constant. According to the same analysis, each time it completes a cycle after stage $s_{1}$, at least $2^{e+3-b_{e}\left[s_{1}\right]}$ additional weight can be counted in the domain of $U$. Alternatively, at least $2^{-b_{e}\left[s_{1}\right]}$ additional weight can be counted in the domain of $N$. Since the weight of the domain of a prefix-free machine is $<1$, after stage $s_{1}$ the marker $T_{\sigma}$ can only move at most $2^{b_{e}\left[s_{1}\right]}$ times. This concludes the induction step.

Notice that 4.2 holds by the explicit action of step (EII) of the construction. Hence it remains to show that the additional strategies succeed in eliminating the $K$-trivial paths from $[T]-[Q]$.

Lemma 5.4. There are no $K$-trivial paths in $[T]-[Q]$.

Proof. It suffices to show that for each $X \in[T]-[Q]$ and for each $e \in \mathbb{N}$ there exists some $i \in \mathbb{N}$ such that $K\left(X \uparrow_{i}\right)>K(i)+e$. Let $\sigma$ be a string of length $\ell_{e}$ such that $T_{\sigma} \subset X$ (where $T_{\sigma}$ refers to the final value of $T_{\sigma}[s]$ ). Also using Lemma 5.3 , let $s_{0}$ be a stage after which level $\ell_{e}$ does not move. This means that $t_{e}[s]$ reaches a limit $t_{e}$ after $s_{0}$. If $K\left(X \uparrow_{t_{e}}\right) \leq K\left(t_{e}\right)+e$, by the choice of $c$ (as a code for machine $N$, see (5.4) ) we would also have $K\left(X \uparrow_{t_{e}}\right)[s] \leq b_{e}[s]+c+e$ at some even stage $s \geq s_{0}$. At that stage the strategy corresponding to $e$ would require attention. Since $X \uparrow_{t_{e}}$ was not enumerated into $Q$, according to Step (EII) of the construction $X \uparrow_{t_{e}}$ was the last extension of $T_{\sigma}[s]$ that was not in $Q$ and thus $T_{\sigma}$ would move. This contradicts the choice of stage $s_{0}$. This contradiction shows that $K\left(X \uparrow_{t_{e}}\right)>K\left(t_{e}\right)+e$.

This concludes the proof of Theorem 1.3 . We note that the ideas that were elaborated in this section can be used in a number of other arguments in order to obtain $\Pi_{1}^{0}$ classes which are free of $K$-trivials. This is especially useful in cases where the crude strategy that is based on 5.1) is not applicable. As an example we mention the main result in Bar10b] about the LK degrees. We say that $A \leq_{L K} B$ if $K^{B}(\sigma) \leq^{+} K^{A}(\sigma)$ for all strings $\sigma$. In other words, if oracle $B$ can compress at least as efficiently as $A$. In Bar10b it was shown that for every $\Delta_{2}^{0}$ set $B$ which is not $K$-trivial, there exist uncountably many sets $X$ such that $X \leq_{L K} B$; in fact, a perfect $\Pi_{1}^{0}$ class of such sequences. Using the strategy that was elaborated in Section 5.1 one can ensure the existence of such a class with no $K$-trivial members. The value of such a strengthening is again the use of basis theorems. For example, by the low for $\Omega$ basis theorem that we discussed in Section 2.2 , we have that for every $\Delta_{2}^{0}$ set $X>_{L K} \emptyset$ there exists a (in fact, uncountably many) low for $\Omega$ set $Z$ such that $\emptyset<_{L K} Z<_{L K} X$.

\section{REFERENCES}

[Bar10a] George Barmpalias. Compactness arguments with effectively closed sets for the study of relative randomness. J. Logic Computation, 2010. In press.

[Bar10b] George Barmpalias. Relative randomness and cardinality. Notre Dame J. Formal Logic, 51(2), 2010.

[BD09] Laurent Bienvenu and Rod Downey. Kolmogorov complexity and Solovay functions. In 26th Annual Symposium on Theoretical Aspects of Computer Science (STACS 2009), pages 147-158. Dagstuhl Seminar Proceedings LIPIcs 3, 2009.

[BLS08] George Barmpalias, Andrew E. M. Lewis, and Frank Stephan. $\Pi_{1}^{0}$ classes, LR degrees and Turing degrees. Ann. Pure Appl. Logic, 156(1):21-38, 2008. 
[BS] George Barmpalias and Tom Sterkenburg. On the number of infinite sequences with trivial initial segment complexity. Preprint, September 2010.

[BV] George Barmpalias and Charlotte Vlek. Kolmogorov complexity of initial segments of sequences and arithmetical definability. Preprint, June 2010.

[Cha76] G. Chaitin. Information-theoretical characterizations of recursive infinite strings. Theoretical Computer Science, 2:45-48, 1976.

[CM06] Barbara F. Csima and Antonio Montalbán. A minimal pair of K-degrees. Proc. Amer. Math. Soc., 134(5):1499-1502 (electronic), 2006.

[DG08] Rod Downey and Noam Greenberg. Turing degrees of reals of positive packing dimension. Information Processing Letters, 108:198-203, 2008.

[DH10] Rod Downey and Denis Hirschfeldt. Algorithmic Randomness and Complexity. Springer-Verlag, 2010.

[DHMn05] Rod Downey, Denis R. Hirschfeldt, Joseph S. Miller, and André Nies. Relativizing Chaitin's halting probability. J. Math. Log., 5(2):167-192, 2005.

[HNS07] Denis R. Hirschfeldt, André Nies, and Frank Stephan. Using random sets as oracles. J. Lond. Math. Soc. (2), 75(3):610-622, 2007.

[Nie09] André Nies. Computability and Randomness. Oxford University Press, 2009.

[RS10] Jan Reimann and Theodore Slaman. Measures and their random reals. Submitted, 2010.

Institute for Logic, Language and Computation, Universiteit van Amsterdam, P.O. Box 94242, 1090 GE Amsterdam, The Netherlands.

E-mail address: barmpalias@gmail.com

$U R L:$ http://www.barmpalias.net/ 